\title{
A Bayesian decision theory approach for the techno-economic analysis of an all-optical router
}

\author{
Víctor López ${ }^{1}$, José Alberto Hernández ${ }^{1}$, Javier Aracil ${ }^{1}$, \\ Juan P. Fernández Palacios ${ }^{2}$ and Óscar González de Dios ${ }^{2}$ \\ 1 Universidad Autónoma de Madrid, Spain \\ [victor.lopez, jose.hernandez, javier.aracil]@uam.es, \\ WWW home page: http://www.ii.uam.es/ networking \\ 2 Telefónica I+D, Spain \\ [jpfpg, ogondio]@tid.es
}

\begin{abstract}
Typically, core networks are provided with both optical and electronic physical layers. However, the interaction between the two layers is at present limited, since most of the traditional transport functionalities, such as traffic engineering, switching and restoration, are carried in the IP/MPLS layer.

In this light, the research community has paid little attention to the potential benefits of the interaction between layers, multilayer capabilities, on attempts to improve the Quality of Service control.

This work shows when to move incoming Label Switched Paths (LSPs) between layers based on a multilayer mechanism that trades off a QoS metric, such as end-to-end delay, and techno-economic aspects. Such mechanism follows the Bayesian decision theory, and is tested with a set of representative case scenarios.
\end{abstract}

\section{Introduction}

Core networks are often provided with both electronic and optical routing capabilities. Essentially, electronic routing has the well-known advantages of statistical multiplexing and granularity, but is a hard-computational process which introduces queuing delay to packets. On the other hand, data packets switched in the optical domain only experience propagation delay. However, optical resources provide a granularity which is too coarse for typical Internet streams, even if they come from the multiplex of many users.

As noted in previous work [1-3], it is highly desirable to efficiently combine the benefits of both optical and electronic domains, according to some policy. In a typical scenario, incoming Label Switched Paths (LSPs) arrive to a multilayercapable router, which has to decide whether to perform optical or electronic switching (fig. 1). If an incoming LSP is routed in the electronic domain, it

* The authors would like to thank the support from the European Union VI Framework Programme e-Photon/ONe+ Network of Excellence (FP6-IST-027497). This work has also been partially funded by the IST Project NOBEL II (FP6-IST-027305). 
suffers hop-by-hop opto-electronic conversion (with subsequent delay), but if it is routed optically a lightpath is reserved end-to-end. Some LSPs share the endto-end lightpath. The choice of electronic or optical switching is based upon a set of previously-defined rules in the multilayer-capable router.

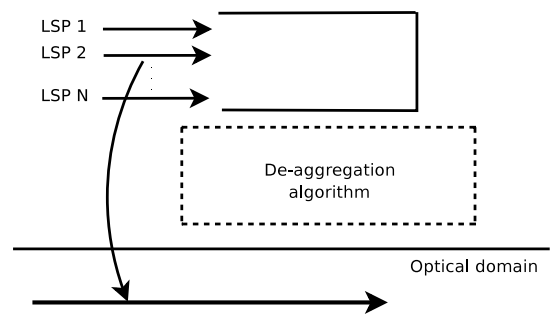

Fig. 1. Multilayer-capable router scenario

In this paper, we propose a techno-economic model to help routers to take the decision of optical or electronic switching of their LSPs. Such approach makes use of Bayesian decision theory, and takes into account several aspects concerning the Quality of Service perceived by packets, by means of queuing delay, and also techno-economic aspects such as the relative cost associated to switching LSPs in either the optical or the electronic domain. The algorithm computation is low cost, because operations are not recursive and only has to be computed when a new LSP crosses the router.

In this light, the remainder of this work is organised as follows: Section 2 covers the mathematical foundations for such techno-economic analysis with a Bayesian decisor. Section 3 provides a set of experiments and numerical examples to show how to reach to an optimal decision. Finally, section 4 outlines a summary of the results obtained and further lines of investigation.

\section{Analysis}

\subsection{Problem statement}

As previously stated, the aim is to define a mathematically rigorous set of rules to help such multilayer-capable core routers decide whether to switch a given LSP in the optical domain or the electronic domain.

At a given time, a multilayer router handles a set of LSPs. The router can switch each LSP either on the electronic domain or the optical domain. Typically, due to QoS constraints, it is preferred optical switching due to the lack of queueing delay. However, optical switching resources are limited (for example, due to wavelength conversion capabilities) and their cost is larger than electronic switching. Thus, the router must trade-off these two parameters: queuing delay versus the cost associated to optical switching, and needs to have a set of rules 
predefined to make a decision on how many LSPs should be switched in the optical domain and how many in the electronic domain.

To do so, let $N$ refer to the number of LSPs handled at a given random time by the multilayer router, and let $L\left(d_{i}, x\right)$ refer to the loss function. The loss function $L\left(d_{i}, x\right)$ denotes the cost or loss of switching $i$ LSPs in the electronic domain (thus, $N-i$ LSPs in the optical domain) with subsequent queueing delay experienced by the packets of the electronically switched LSPs, which is denoted $x$ (for simplicity, the optically switched LSPs have been assumed to experience zero delay). The term $d_{i}$ denotes the "decision" of routing $i$ LSPs out of a total of $N$ in the electronic domain, and is defined for some decision space $\Omega=\left\{d_{1}, \ldots, d_{N}\right\}$. In this light, $L\left(d_{i}, x\right)$ is given by:

$$
L\left(d_{i}, x\right)=\left(C_{e}(i)+C_{o}(N-i)\right)-U(x), \quad i=1, \ldots, N, \quad x>0
$$

where $C_{e}(i)$ and $C_{o}(N-i)$ refer to the cost associated to routing $i$ LSPs in the electronic domain and $U(x)$ refers to the utility associated to a queuing delay of $x$ units of time, experienced by the electronically switched LSPs.

Following [4], the Bayes risk, which is essentially the expectation of the loss function with respect to $x$, equals:

$$
R\left(d_{i}\right)=\mathbb{E}_{x} L\left(d_{i}, x\right)=\left(C_{e}(i)+C_{o}(N-i)\right)-\mathbb{E}_{x} U(x), \quad i=1, \ldots, N
$$

The goal is to obtain the optimal decision $d^{*}$ such that the Bayes risk $R\left(d^{*}\right)$ is minimum. In other words:

$$
\text { find } d^{*} \text { such that } R\left(d^{*}\right)=\min _{d_{i}, i=1, \ldots, N} R\left(d_{i}\right)
$$

The next section proposes a set of utility functions, $U(x)$, that measures the QoS experienced (in terms of queuing delay) by the electronically-switched packets; and also introduces a metric for quantifying the relative cost of optical switching with respect to electronic switching.

\subsection{The utility function $U(x)$}

As previously stated, the utility function $U(x)$ is defined over the random variable $x$, which represents the queuing delay experienced by the packets of electronically switched LSPs. The queuing delay shall be assumed to be Weibull distributed, since this has been shown to accurately capture the queueing delay behaviour of a router with self-similar input traffic [5-7]. In this light, the delay probability density function is given by [5]:

$$
p(x)=(2-2 H) C \frac{(C-m)^{2 H}}{2 K(H)^{2} a m}(C x)^{1-2 H} \exp \left(-\frac{(C-m)^{2 H}}{2 K(H)^{2} a m}(C x)^{2-2 H}\right), \quad x>0
$$


where $C$ is the lightpath capacity, $m$ is the average input traffic and $a$ is a variance coefficient such that $a m=\sigma^{2}$ (with $\sigma^{2}$ being the input traffic variance) and $H$ is the Hurst parameter.

Once $p(x)$ has been defined, the next step is to define a measure of the "utility" associated to routing LSPs in the electronic domain.

Delay based utility In its simplest way, we can easily evaluate the utility based on the observed delay, that is, $U_{\text {delay }}(x)=-x$. The utility function is thus opposite to the queuing delay $x$, since the more utility occurs for smaller delays. Thus, computing the Bayes risk defined in eq. 2 yields:

$$
E_{x}\left[U_{\text {delay }}(x)\right]=E_{x}[-x]=-\int_{0}^{\infty} x p(x) d x
$$

which equals the average queuing delay experienced by the electronically-switched packets. Such value takes the following analytical expression:

$$
E_{x}\left[U_{\text {delay }}(x)\right]=-\frac{1}{C}\left(\frac{(C-m i)^{2 H}}{2 K(H)^{2} a m i}\right)^{1 /(2-2 H)} \Gamma\left(\frac{3-2 H}{2-2 H}\right)
$$

However, the average delay is not always a useful (or at least, representative) metric in the evaluation of the Quality of Service experienced by certain applications, especially when quantifying the relative QoS experienced by realtime applications. The following considers two other utility functions used in the literature for hard-real time and elastic applications $[8,9]$.

Hard real-time utility Hard real-time applications are those which tolerate a delay of up to a certain value, say $T_{\max }$, but their performance degrades very significantly when the delay exceeds such value. Examples are: online gaming, back-up services and grid applications. The parameter $T_{\max }$ denotes the tolerated delay threshold for each particular application. The ITU-T recommendation Y.1541 [10] and the 3GPP recommendation S.R0035 [11] defined service classes based on thresholds.
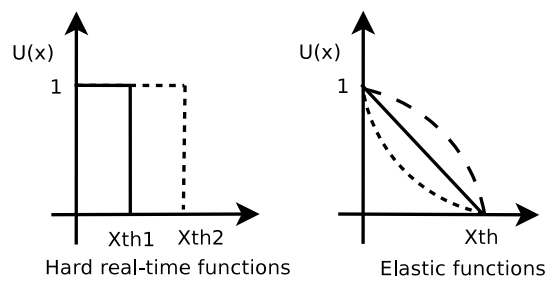

Fig. 2. Utility functions: hard real-time (left) and elastic (right)

Hard real-time utility can thus be modelled by a step function as shown in figure 2 left, and takes the expression: 


$$
U_{\text {step }}(x)= \begin{cases}1 & \text { if } x<x_{p} \\ 0 & \text { otherwise }\end{cases}
$$

To compute the Bayes risk requires the average utility:

$$
E_{x}\left[U_{\text {step }}(x)\right]=\int_{0}^{T_{\max }} p(x) d x=1-\int_{T_{\max }}^{\infty} p(x) d x=1-\mathbb{P}\left(x>T_{\max }\right)
$$

which, according to 3 , leads to:

$$
E_{x}\left[U_{\text {step }}(x)\right] \sim 1-\exp \left(-\frac{(C-m i)^{2 H}}{2 K(H)^{2} a m i}(C x)^{2-2 H}\right), \quad x>0
$$

Elastic utility Other services consider a more flexible QoS function, since the service is degraded little by little (fig. 2 right). These services considers zero delay as the maximum possible utility, but the utility reduces with increasing delay. The ITU-T recommendation G.107 defines the "E model" [12], which explains in detail the degradation of voice service in humans. In other utility function studios, the exponential function has been used to describe the degradation of elastic services [9].

Thus, the elastic utility function is modelled as:

$$
U_{\exp }(x)=\lambda e^{-\lambda x}, \quad x>0
$$

where $\lambda$ refers to decay ratio of the exponential function. Following the definition of $T_{\max }$ above, the value of $\lambda$ has been chosen such that $90 \%$ of the utility lies before $T_{\max }$. That is:

$$
\lambda=\frac{1}{T_{\max } \log (1-0.9)}
$$

Finally, the average elastic utility follows:

$$
E_{x}\left[U_{\exp }(x)\right]=\int_{0}^{\infty} \lambda e^{-\lambda x} p(x) d x
$$

which has no analytical form. However, we can use the Taylor expansion to approximate it, since:

$$
\begin{aligned}
E[f(x)] & \approx \int_{0}^{\infty} p(x)\left(f(E[x])+f^{\prime}(E[x])(x-E[x])+\frac{1}{2} f^{\prime \prime}(E[x])(E[x]-x)^{2}\right) d x \\
& =f(E[x])+\frac{1}{2} f^{\prime \prime}(E[x]) \sigma_{x}^{2}
\end{aligned}
$$

Thus:

$$
E_{x}\left[U_{\exp }(x)\right] \approx U_{\exp }\left(E_{x}[x]\right)+\frac{1}{2} U_{\exp }^{\prime \prime}\left(E_{x}[x]\right) \sigma_{x}^{2}
$$


where the variance $\sigma_{x}^{2}$ can be easily derived from eq. 3:

$$
\sigma_{x}^{2}=\frac{1}{C^{2}}\left(\frac{2 K(H)^{2} a m i}{(C-m i)^{2 H}}\right)^{1 /(1-H)}\left(\Gamma\left(\frac{2-H}{1-H}\right)+\Gamma^{2}\left(\frac{3-2 H}{2-2 H}\right)\right)
$$

\subsection{The economic cost of electronic and optical switching}

As previously stated, the values of $C_{e}(i)$ and $C_{o}(N-i)$ in eq. 1 represent the cost associated to switching $i$ LSPs in the electronic domain and $N-i$ in the optical domain. Typically, the optical resources are more precious than the electronic resources, hence we will penalise the optical switching more than electronic switching.

For simplicity purposes, we have considered a linear cost approach, at which electronic switching is penalised as $C_{e}(i)=K i$ for some $K>0$, and the cost of optical switching is $C_{o}(N-i)=R_{\text {cost }} K(N-i)$. The value of $R_{\text {cost }}$ denotes the relative optical-electronic cost, that is, the ratio at which the optical cost increases with respect to the electronic cost.

\section{Experiments and results}

This section provides a few numerical examples applied to real case scenarios. The aim is to show a few practical cases at which the implemented algorithm at a given core multilayer switch decides the number of optically-switched LSPs that should be transmitted according to three sets of parameters: (1) QoS parameters, essentially the $T_{\max }$ value introduced above; $(2)$ the relative cost $R_{\text {cost }}$ which provides a measure of the economic cost of switching LSPs in the optical domain with respect to the electronic switching; and, (3) the self-similar characteristics of the incoming flows, represented by the Hurst parameter $H$.

The simulation scenario assumes a 2.5 Gbps core network, which carries a number of $N=72$ standard VC-3 LSPs (typically $34.358 \mathrm{Mbps}$ each). The values of $m, \sigma$ and $H$, which represent the characteristics of the traffic flows, i.e. average traffic load, variability and Hurst parameter, have been chosen as $H=0.6$ (according to [13]) and $m$ and $\sigma$ such that $\frac{\sigma}{m}=0.3$.

Finally, the value of $K$ has been chosen as $K=\frac{1}{N}$, in order to get the electrical cost normalised, i.e. within the range $[0,1]$.

\subsection{Study of threshold $T_{\max }$}

This experiment shows the influence of the choice of $T_{\max }$ in the decision to be made by the multi-layer router. Fig. 3 shows this case for several values of $T_{\max }$ assuming the step utility function (left) and the exponential utility function (right). The values of $T_{\max }$ have been chosen to cover a wide range from $0.1 \mathrm{~ms}$ to $100 \mathrm{~ms}$. Clearly, the number of optically-switched LSPs should increase with increasing values of $T_{\max }$, since high QoS constrains require small delays in the 

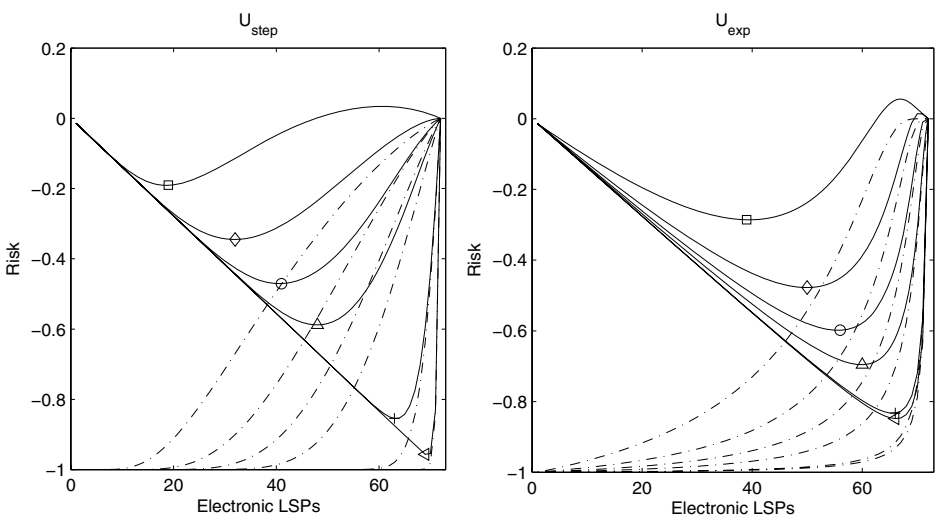

$T_{\max }=0.1 \mathrm{~ms} \diamond T_{\max }=0.25 \mathrm{~ms} \quad \circ \quad T_{\text {max }}=0.5 \mathrm{~ms}$

$\triangle T_{\max }=1 \mathrm{~ms}+T_{\max }=10 \mathrm{~ms} \triangleleft \mathrm{T}_{\max }=100 \mathrm{~ms}$

Fig. 3. Optimal decisions for several $T_{\max }$ values assuming hard real-time utility function (left) and elastic utility function (right). Dashed line = Utility.

packet transmission (thus larger number of optically-switched LSPs to reduce latency).

Typically, most of the end-to-end delay suffered by applications occur in the access network, and it is widely accepted that the core network should be designed to introduce delay of no more than $1-10 \%$ of the total end-to-end delay. For hard real-time applications, which may demand a maximum end-to-end of $100 \mathrm{~ms}$, the core delay is thus in the range of $1-10 \mathrm{~ms}$, which implies a total number of electronically-switched LSPs of $d^{*}=d_{48}$ and $d^{*}=d_{63}$ respectively of a total of $N=72$ LSPs. For the same delay constrains, elastic applications impose a number of electronically-switched LSPs of $d^{*}=d_{60}$ and $d^{*}=d_{66}$ respectively.

Obviously, the delay requirements for hard real-time applications are tighter than those for elastic applications, thus demanding a larger number of opticallyswitched LSPs, as shown in fig. 3.

\subsection{Analysis with different $\boldsymbol{R}_{\text {cost }}$ values}

This experiment shows the impact of the relative cost $R_{\text {cost }}$, which refers to the relative cost of optical switching with respect to electronic switching, in the final decision $d^{*}$, to be taken by the multilayer router. Fig. 4 left shows where the optimal decision lies (minimum cost) for different $R_{\text {cost }}$ values considering the case of mean utility function $\left(T_{\max }=10 \mathrm{~ms}\right)$. As shown, the more expensive optical switching is (large values of $R_{\text {cost }}$ ), the less number of LSPs are switched optically.

Fig. 4 right shows where the optimal decision lies considering the mean (dashed), exponential (dotted) and step (solid) utility functions, for different values of $R_{\text {cost }}$. Again, as the $R_{\text {cost }}$ value increases, the number of opticallyswitched LSPs decreases (hence, larger number of LSPs switched in the electronic 

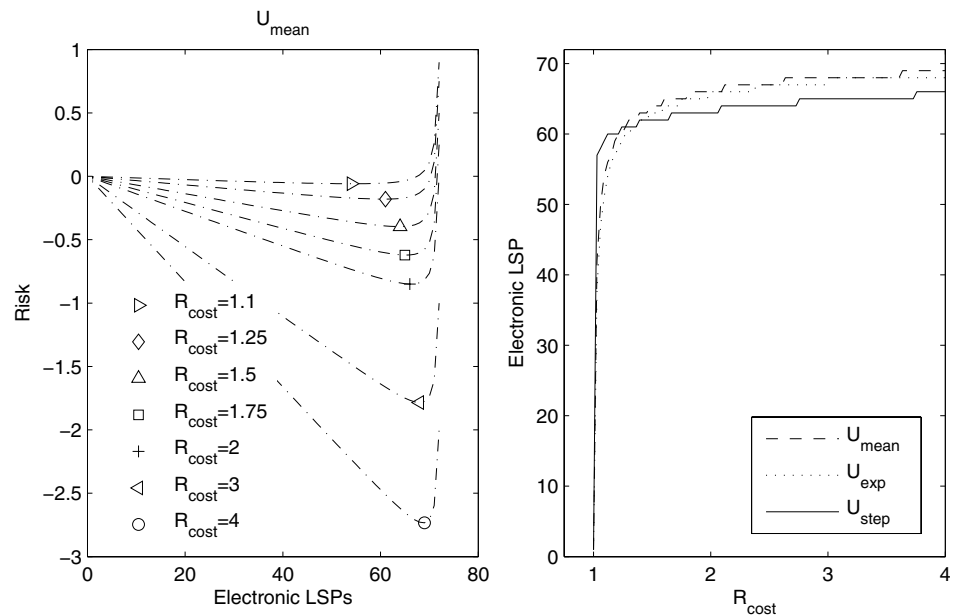

Fig. 4. Study of relative cost $\left(R_{\text {cost }}\right)$ variation

domain). As shown, the step utility function, which represents the highest QoS constraints, demands more optically-switched LSPs than the other two, for large values of $R_{\text {cost }}$.

Finally, it is worth noticing that, for values $R_{\text {cost }} \geq 1.5$, the optimal decision lies in the range $d_{65}$ to $d_{72}$. The reason for this is that, when optical switching becomes too expensive, the $R_{\text {cost }}$ is critical in the optimal decision, thus cancelling any influence of the QoS parameter $T_{\max }$. In this light, the network operator has a means to decide where the optimal decision lies, trading off the $R_{\text {cost }}$ parameter and the QoS values.

\subsection{Influence of the Hurst parameter $H$}

The previous two numerical examples have assumed a value of $H=0.6$, as observed in real backbone traces [13]. However, other scenarios may show different values of $H$ and it is interesting to study its impact on the bayesian decisor. In this light, fig. 5 shows the influence (left) or no-influence (right) of such parameter $H$ in the optimal decision. In spite that long-range dependence degrades queuing performance generally, at high-delay values, the delay variability is smaller for high values of $H$ (see [5], fig. 5).

Thus, the characteristics of the incoming traffic have a more or less impact on the bayesian decisor, depending on the QoS parameters. When $T_{\max } \geq 10 \mathrm{~ms}$, there is little influence of $H$ (fig 5 right), but for $T_{\max }=1 \mathrm{~ms}$ and smaller, the value of $H$ is key since it moves the decision in a wide range of optimal values: from $d_{31}$ in the case of $H=0.5$ to $d_{64}$ for $H=0.9$ (fig 5 left).

The level curves shown in fig. 6 shows such behaviour for the three utility functions $\left(T_{\max }=1 \mathrm{~ms}\right)$. Each level curve corresponds to a different utility. Fig. 6 left (case of mean utility function) should read as no influence with the 

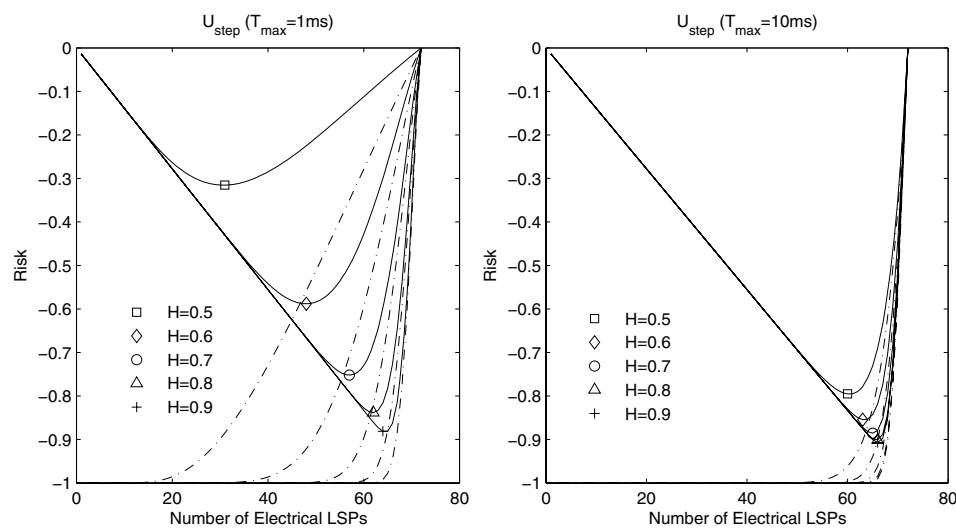

Fig. 5. Hurst parameter variation

Hurst parameter (i.e. parallel level curves $=$ optimal decision independent of $H$ value). On the contrary, fig. 6 middle (case of exponential utility function) and right (case of step utility function) shows more influence with the $H$ value (i.e. less parallelness in the level curves).
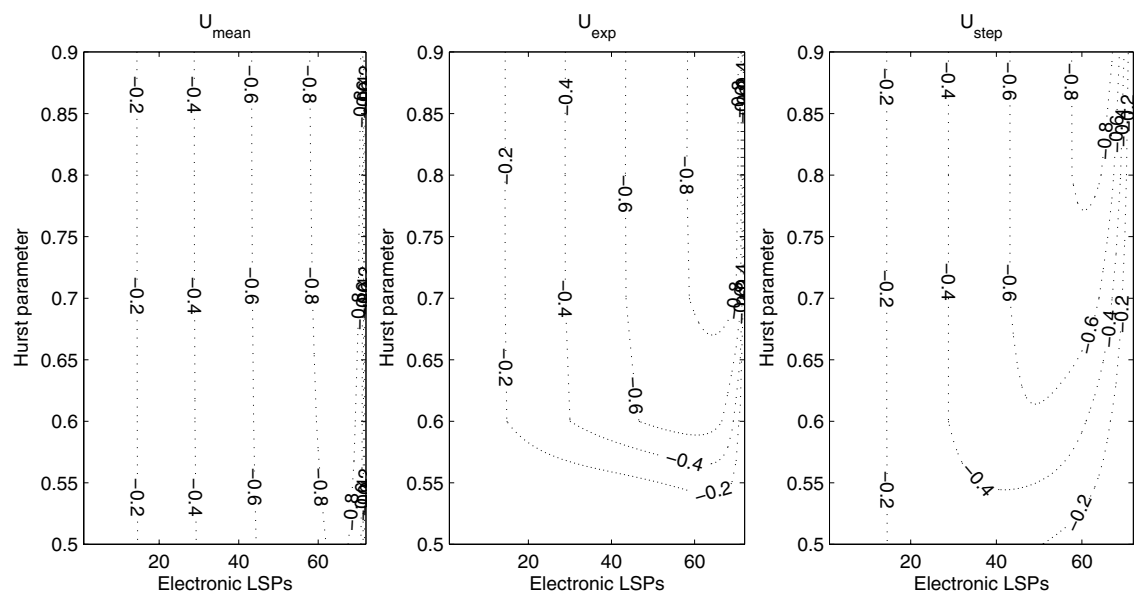

Fig. 6. Hurst parameter variation

\section{Summary and conclusions}

This work's main contribution is two-fold: First, it presents a novel methodology, based on the Bayesian decision theory, that helps multilayer-capable routers to 
take the decision of either optical or electronic switching of incoming LSPs. Such decision is made based on technical aspects such as QoS constrains and long-range dependence characteristics of the incoming traffic, but also considers the economic differences of optical and electrical switching. This way permits high flexibility to the network operator to trade off both economic and technical aspects.

The algorithm proposed is of low complexity, and can easily adapt to changing conditions: QoS guarantees, traffic profiles, economic conditions and network operator preferences.

This algorithm can be implemented in a per node basis by using local and independent parameters (e.g delay thresholds) in each node. However, in further extensions of this mechanism, the local parameters used in each node will be based on information about end-to-end delay through the whole network.

\section{References}

1. Sato, K., Yamanaka, N., Takigawa, Y., Koga, M., Okamoto, S., Shiomoto, K., Oki, E., Imajuku, W.: GMPLS-based photonic multilayer router (Hikari router) architecture: an overview of traffic engineering and signaling technology. IEEE Communications Magazine 40(3) (2002) 96-101

2. Puype, B., Yan, Q., De Maesschalck, S., Colle, D., Pickavet, M., Demeester, P.: Optical cost metrics in multi-layer traffic engineering for IP-over-optical networks. In: Transparent Optical Networks, 2004. Proceedings of 2004 6th International Conference on. Volume 1. (2004) 75-80

3. Vigoureux, M., Berde, B., Andersson, L., Cinkler, T., Levrau, L., Ondata, M., Colle, D., Fernandez-Palacios, J., Jager, M.: Multilayer traffic engineering for GMPLS-enabled networks. IEEE Communications Magazine 43(7) (2005) 44-50

4. French, S., Ríos Insúa, D.: Statistical decision theory. Oxford University Press Inc. (2000)

5. Norros, I.: On the use of fractional Brownian motion in the theory of connectionless networks. IEEE J. Selected Areas in Communications 13(6) (1995) 953-962

6. Papagiannaki, K., Moon, S., Fraleigh, C., Thiran, P., Diot, C.: Measurement and analysis of single-hop delay on an ip backbone network. IEEE J. Selected Areas in Communications 21(6) (2003) 908-921

7. Hernández, J.A., Phillips, I.W.: Weibull mixture model to characterise end-to-end Internet delay at coarse time-scales. IEE Proc. Communications 153(2) (2005)

8. Shenker, S.: Fundamental design issues for the future internet. IEEE Journal on Selected Areas in Communications 13(7) (1995) 1176-1188

9. Choi, Y.J., Bahk, S.: Qos scheduling for multimedia traffic in packet data cellular networks. In: IEEE International Conference on Communications, 2003. Volume 1. (2003) 358-362

10. ITU-T: ITU-T Recommendation Y.1541 - Network Performance Objectives for IP-Based Services (2003)

11. 3GPP: 3GPP Recommendation s.r0035-0 v1.0. - Quality of Service (2002)

12. ITU-T: ITU-T Recommendation G.107 : The E-model, a computational model for use in transmission plannings (2005)

13. Clegg, R.G.: Markov-modulated on/off processes for long-range dependent internet traffic. ArXiv Computer Science e-prints (2006) 